\title{
Anti-Inflammatory, Anti-Angiogenic and Anti-Nociceptive Activities of 4-Hydroxybenzaldehyde
}

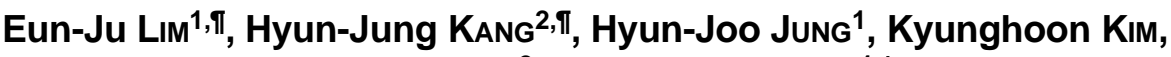 \\ Chang-Jin LIM ${ }^{2}$ and Eun-Hee PARK, ${ }^{1, *}$ \\ 'College of Pharmacy, Sookmyung Women's University, Seoul 150-742, Korea \\ ${ }^{2}$ Division of Life Sciences and Research Institute of Life Sciences, Kangwon National University, Chuncheon 200-701, Korea
}

(Received July 21, 2008; Revised September 2, 2008; Accepted September 3, 2008)

\begin{abstract}
The current work was designed to assess novel pharmacological activities of 4-hydroxybenzaldehyde (HD), a major phenolic constituent of various natural products of plant origin, such as Gastrodia elata Blume. HD exhibited a significant inhibition in the chick chorioallantoic membrane (CAM) angiogenesis. HD also displayed an inhibitory effect in acetic acid-induced permeability in mice. Anti-nociceptive activity of HD was convinced using the acetic acid-induced writhing test in mice. HD was able to suppress production of nitric oxide (NO) and induction of inducible nitric oxide synthase (iNOS) and cyclooxygenase-2 (COX-2) in the lipopolysaccharide (LPS)-activated RAW264.7 macrophages. HD also diminished the reactive oxygen species (ROS) level elevated in the LPS-activated macrophages. In brief, HD exhibits anti-angiogenic, anti-inflammatory and anti-nociceptive activities possibly via down-regulating iNOS and/or COX-2, which may be partly responsible for pharmacological efficacies of various natural products.
\end{abstract}

Keywords: Anti-angiogenic, Anti-inflammatory, Anti-nociceptive, Cyclooxygenase-2, 4-hydroxybenzaldehyde, Inducible nitric oxide synthase, Nitric oxide, RAW264.7, Reactive oxygen species

4-Hydroxybenzaldehyde (HD, Fig. 1) is one of the major phenolic constituents of various natural products used in traditional medicine, such as Gastrodia elata Blume (Ha et al., 2001) in the family of Orchidaceae. G elata has been widely used for the treatment of head-<smiles>O=Cc1ccc(O)cc1</smiles>

4-Hydroxybenzaldehyde (HD)

Fig. 1. Chemical structure of 4-hydroxybenzaldehude (HD), a major phenolic compound of plant origin.

${ }^{*}$ Corresponding author

Tel: $+82-2-710-9565$, fax: $+82-2-3273-5847$

E-mail: ehpark@sookmyung.ac.kr ache, tetanus, rheumatism and convulsive disorders in oriental countries, such as Korea, China and Japan. HD was shown to significantly inhibit $\gamma$-aminobutyric acid (GABA) transaminase via enhancement of the binding of endogenous receptor agonist in the presence of GABA, a major inhibitory neurotransmitter in the mammalian brain (Ha et al., 2000; Ha et al., 2001). HD contains anxiolyticlike effect via the GABAnergic nervous system (Jung et al., 2006). Recently, it has been shown that HD is able to protect against ischemic neuronal cell death in the hippocampal CA1 region (Kim et al., 2007). However, antiinflammatory and related activities of HD have not been assessed in the aspect of pharmacology so far. In this work, we demonstrate that HD possesses anti-angiogenic, anti-inflammatory and anti-nociceptive activities using both in vivo and in vitro experimental models..

\section{MATERIALS AND METHODS}

\section{Chemicals, fertilized eggs and experimental animals}

4-Hydroxybenzaldehyde (HD), Evans blue, E. coli lipopolysaccharide (LPS) and aminopyrine were obtained from Sigma Chemical Co. (St. Louis, MI, USA). 2',7'- 
Dichlorodihydrofluorescein diacetate (DCFH-DA) was purchased from Invitrogen (Carlsbad, CA, USA). All other chemicals used were of reagent grade or better. Fertilized brown Leghorn eggs were obtained from Pulmuone Food Co., Seoul, Korea. Male ICR mice (male, 4-weekold, about $25 \mathrm{~g}$ ) were ordered from Samtaco Animal Farm, Osan, Korea, and acclimated for one week prior to treatment. The animal room was maintained at $23 \pm 2^{\circ} \mathrm{C}$ with a 12-h light/dark cycle. Food and tap water were supplied ad libitum. The ethical guidelines, described in the NIH Guide for Care and Use of Laboratory Animals, were followed throughout the experiments.

\section{Cell culture}

The RAW264.7 cells, a murine macrophage cell line, were from American Type Culture Collection (Manassas, VA, USA). The mammalian cells were grown in Dulbecco's modified Eagle's medium containing 10\% heatinactivated fetal bovine serum (FBS), $25 \mathrm{mM} \mathrm{HEPES} \mathrm{(pH}$ 7.5), $100 \mathrm{U} / \mathrm{ml}$ penicillin and $100 \mu \mathrm{g} / \mathrm{ml}$ streptomycin. The macrophage cells were maintained in a humidified atmosphere containing $5 \% \mathrm{CO}_{2}$, plated at a density of $1 \times 10^{6}$ and preincubated for $24 \mathrm{~h}$ at $37^{\circ} \mathrm{C}$. For all experiments, the macrophage cells were grown to $80-90 \%$ confluence, and subjected to no more than 20 cell passages.

\section{Chorioallantoic membrane (CAM) assay}

Anti-angiogenic activity was determined using CAM assay (Song et al., 2003) as followed. The fertilized eggs were kept at $37^{\circ} \mathrm{C}$ in a humidified egg incubator. After 3.5-day incubation, about $2 \mathrm{ml}$ of albumen was aspirated from the eggs through the small hole drilled at the narrow end, permitting the small CAM and yolk sac to drop away from the shell membrane. The shell covering the air sac was punched out and removed by forceps, and the shell membrane on the floor of the air sac was peeled away. In the 4.5-day-old chick embryo, an HD-loaded Thermanox coverslip was applied onto the CAM surface. Two days after returning the chick embryo to the incubator, an appropriate volume of $10 \%$ fat emulsion (Intralipose, $10 \%$ ) was injected into a 6.5-day-old embryo chorioallantois. Using a score system under a microscope, the branching pattern of each egg was graded as $0,1+$ or $2+$. Convergence of a few vessels toward the CAM surface was denoted as 1+, and 2+ reflected an increased density and length of vessels toward the CAM surface.

\section{Acetic acid-induced vascular permeability test}

According to a modification of the method of Whittle (1964), acetic acid-induced vascular permeability test was performed to assess anti-inflammatory activity of HD. One hour after oral administration of HD (50 or $100 \mathrm{mg} / \mathrm{kg}$ ) or aminopyrine (AP, $100 \mathrm{mg} / \mathrm{kg}$ ), $0.1 \mathrm{ml} / 10 \mathrm{~g}$ body weight of $1 \%$ Evans blue solution was injected intravenously in each mouse. Thirty min later, $0.1 \mathrm{ml} / 10 \mathrm{~g}$ body weight of $0.7 \%$ acetic acid in saline was intraperitoneally injected. Thirty min after the administration of acetic acid, the mice were sacrificed by cervical dislocation. After $10 \mathrm{ml}$ of saline was injected into the peritoneal cavity, the washing solutions were collected in test tubes. The concentration of Evans blue in the washing solution was spectrophometrically determined by the absorbance at $630 \mathrm{~nm}$. The vascular permeability was represented in terms of the absorbance $\left(A_{630}\right)$ which leaked into the peritoneal cavity.

\section{Acetic acid-induced writhing response}

An anti-nociceptive activity of HD was assessed as previously described (Olajide et al., 2000). The response to an intraperitoneal injection of acetic acid solution into mice, manifesting as a contraction of the abdominal muscles and stretching of hind limbs, was evaluated. Nociception was induced by intraperitoneal injection of $0.7 \%$ acetic acid solution at the dose of $0.1 \mathrm{ml} / 10 \mathrm{~g}$ body weight. Each experimental group was orally treated with vehicle (saline), HD (50 or $100 \mathrm{mg} / \mathrm{kg}$ ) or aminopyrine (AP, 100 $\mathrm{mg} / \mathrm{kg}$ ) as a positive control. From 10 min later, the number of writhes during the following 10 min was counted.

\section{Nitrite analysis}

Accumulated nitrite $\left(\mathrm{NO}_{2}^{-}\right)$in the culture media was determined according to a colorimetric assay based on the Griess reaction (Sherman et al., 1993). The supernatants $(100 \mu \mathrm{l})$, obtained after centrifugation, were mixed with $100 \mu \mathrm{l}$ Griess reagent $(6 \mathrm{mg} / \mathrm{ml})$ at room temperature for $10 \mathrm{~min}$, and then $\mathrm{NO}_{2}{ }^{-}$concentration was determined by the absorbance at $540 \mathrm{~nm}$. The calibration curve was constructed using the known concentrations of sodium nitrite.

\section{Determination of intracellular ROS}

For determination of intracellular ROS level, the redoxsensitive fluorescent probe DCFH-DA was used as previously described (Royall and Ischiropoulos, 1993). After preincubation with $\mathrm{HD}(0,0.5,1.0$ or $2.5 \mathrm{mM})$ or vehicle (saline) for $1 \mathrm{~h}$, the RAW264.7 cells were treated with LPS for $24 \mathrm{~h}$. Afterwards, they were incubated with $5 \mu \mathrm{M}$ DCFH-DA for $30 \mathrm{~min}$ at $37^{\circ} \mathrm{C}$. The harvested cells were imediately analyzed by a flow cytometry.

\section{Immunoblot analysis}

The RAW264.7 macrophage cells were treated with 
LPS $(1 \mu \mathrm{g} / \mathrm{ml})$ in the presence or absence of HD for $24 \mathrm{~h}$ and then washed twice with ice-cold phosphate-buffered saline (PBS). The mammalian cells were lysed in a buffer containing $20 \mathrm{mM}$ HEPES (pH 7.9), $0.1 \mathrm{M} \mathrm{KCl}, 0.3 \mathrm{M}$ $\mathrm{NaCl}, 10 \mathrm{mM}$ EDTA, $1 \%$ SDS, $1 \mathrm{mM}$ PMSF, $1 \mu \mathrm{g} / \mathrm{ml}$ leupeptin and $1 \mu \mathrm{g} / \mathrm{ml}$ pepstatin. For immunoblotting, antiinducible nitric oxide synthase (anti-iNOS; Transduction Laboratories, Lexington, KY, USA), anti-cyclooxygenase2 (anti-COX-2; Transduction Laboratories, Lexington, KY, USA) and anti- $\beta$-actin (Sigma-Aldrich, St. Louis, MO, USA) antibodies were used.

\section{Statistical analysis}

The data were analyzed for statistical significance using Student's $t$-test. $P$ values less than 0.05 were considered to be significant.

\section{RESULTS AND DISCUSSION}

Down-regulation of angiogenesis is believed to be helpful for prevention of neoplastic growth and inflammation. In reality, some anti-angiogenic substances are known to be effective in animal models of arthritis, whereas several antirheumatic drugs, such as indomethacin, methotrexate and corticosteroids, contain anti-angiogenic activity (Tong et al., 2004). Accordingly, many researchers have been trying to screen useful anti-angiogenic principles from various natural resources. The CAM assay, using an extra-embryonic membrane commonly used in vivo to measure both angiogenesis and anti-angiogenesis, was used for assessing the inhibitory activity of HD on vascular development, and retinoic acid was used as a positive control for the assay. The disk only could't cause any changes in vascular density, indicating that it was unable to influence the formation of blood vessels in the CAM assay (data not shown). After the 2-day treatment, retinoic acid at $1 \mu \mathrm{g} / \mathrm{egg}$ gave rise to an inhibition of $81.3 \%$ in the branching patterns of blood vessels (Fig. 2). When $0.3,1.0$ and $3.0 \mu \mathrm{g} / \mathrm{egg}$ of $\mathrm{HD}$ was applied in the CAM assay, the inhibition percentages in the CAM angiogenesis were $36.3 \%, 55.2 \%$ and $64.9 \%$, respectively (Fig. 2). This clearly indicates that HD exerts an anti-angiogenic activity in a dose-dependent manner. The dose required for half-maximal inhibition $\left(\mathrm{IC}_{50}\right)$ of $\mathrm{HD}$ was calculated to be $1.21 \mu \mathrm{g} / \mathrm{egg}$. In brief, HD contains an anti-angiogencic activity, providing a pharmacological basis on the traditional use of various folkloric medicines for the treatment of inflammatory diseases and cancer.

In a vascular permeability test, a model typical of the first stage inflammatory reactions, inflammatory media-

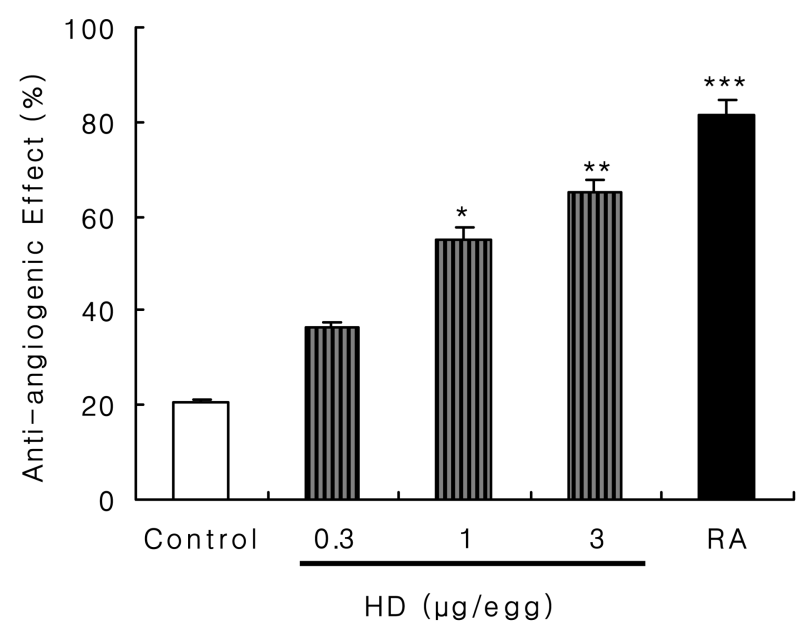

Fig. 2. Dose-dependent anti-angiogenic activity of HD in the chick embryo chorioallantoic membrane (CAM) assay. Retinoic acid (RA, $1 \mu \mathrm{g} / \mathrm{egg}$ ) was used as a positive control. Each column represents mean $\pm \mathrm{S}$.E.

${ }^{\star}, P<0.05 ;{ }^{* *}, P<0.01 ;{ }^{* * *}, P<0.001$.

tors, released following stimulation, leads to dilation of blood vessels and enhanced vascular permeability (Vogel and Vogel, 1997). HD at the oral doses of 50 and 100 $\mathrm{mg} / \mathrm{kg}$ exerted an inhibition of 10.9 and $36.2 \%$ on vascular permeability, respectively (Fig. 3). An inhibitory effect of $\mathrm{HD}$ at $100 \mathrm{mg} / \mathrm{kg}$ is comparable to that of aminopyrine $(100 \mathrm{mg} / \mathrm{kg}$ ) used as a positive control (Fig. 3). Collectively, it is assumed that an acute inflammatory activity of $\mathrm{HD}$ results from its prevention on the release of inflam-

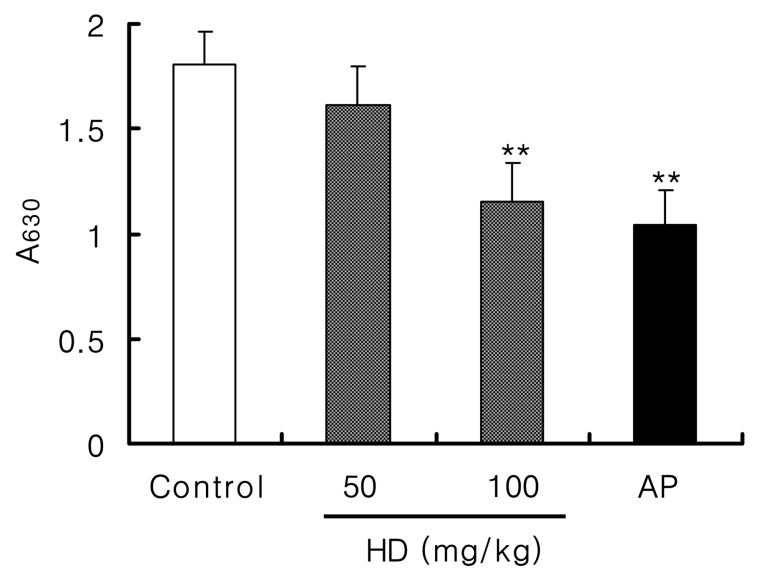

Fig. 3. Inhibitory effect of HD in acetic acid-induced vascular permeability in mice. HD (50 and $100 \mathrm{mg} / \mathrm{kg}$ ) was orally administered. Aminopyrine (AP, $100 \mathrm{mg} / \mathrm{kg}$ body weight) was used as a positive control. Each experimental group contained 7 mice. Vascular permeability was represented by the absorbance at $630 \mathrm{~nm}$. Each column represents mean \pm S.E. ${ }^{* *}, P<0.01$. 
matory mediators at the first stage.

In the acetic acid -induced writhing response, the antinociceptive mechanism of abdominal writhing induced by acetic acid involves the release of arachidonic acid metabolites via cyclooxygenase, and prostaglandin biosynthesis (Franzotti et al., 2002). As shown in Fig. 4, HD at 50 and $100 \mathrm{mg} / \mathrm{kg}$, p.o., gave rise to an inhibition by $27.2 \%$ and $41.0 \%$, respectively, on the writhing response induced by acetic acid. This finding indicates that HD contains anti-nociceptive activity in addition to anti-inflammatory activity, subsequently proposing that prostaglandin biosynthesis might be commonly involved in the pharmacological activities of HD.

Nitric oxide (NO), one of important proinflammatory mediators in the pathogenesis of inflammation, is synthesized from L-arginine by the three isoforms of nitric oxide synthase (NOS), such as neuronal NOS (nNOS), endothelial NOS (eNOS) and inducible NOS (iNOS). For expression of inducible NOS (iNOS), the mammalian cells should be experimentally triggered by specific stimulants such as pro-inflammatory cytokines and bacterial LPS (Chesrown et al., 1994). Since iNOS-derived NO plays a crucial role in various pathological conditions such as inflammation and autoimmune diseases and leads to cellular injury (Singh et al., 2000), suppression of iNOS is closely linked with anti-inflammatory action. Inhibitory effects of HD was tested on LPS-induced NO production in the RAW264.7 macrophages (Fig. 5). Level of the accumulated nitrite, detected by the Griess method, in the culture media was used as an index for NO. After treatment with LPS, nitrite level markedly

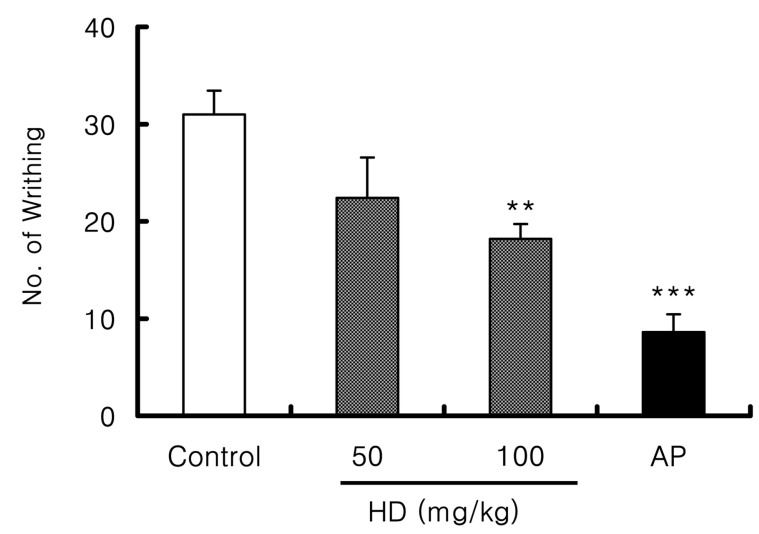

Fig. 4. Inhibitory effect of HD in acetic acid-induced writhing response in mice. HD (50 and $100 \mathrm{mg} / \mathrm{kg}$ ) was orally administered. Aminopyrine (AP, $100 \mathrm{mg} / \mathrm{kg}$ body weight) was used as a positive control. Each experimental group contained 7 mice. Experiments were performed in triplicate. Each column represents mean \pm S.E. ${ }^{* *}, P<0.01 ;{ }^{* * *}, P<0.001$. increased about 7.6-fold. When the macrophage cells were treated with $0.5,1.0$ and $2.5 \mathrm{mM} \mathrm{HD}$, NO production induced by LPS was significantly suppressed in a dose-dependent manner (Fig. 5). No cytotoxic effects of HD on the RAW264.7 macrophages were observed at the used concentrations, which was determined by the MTT assay (data not shown). With the assumption that suppression by HD of NO induction might be caused by a decrease in the iNOS level, the effect of HD on the iNOS expression was tested in the macrophages cells treated with LPS. As shown in Fig. 6, HD dose-dependently suppressed iNOS induction without changes in the levels of $\beta$-actin, an internal control. This result suggests that HD is able to specifically inhibit iNOS induction in the LPSstimulated macrophage cells. Non-steroidal anti-inflammatory drugs (NSAIDs), very beneficial in the treatment of various inflammatory disorders, target cyclooxygenase (COX), a rate-limiting enzyme involved in the conversion of arachidonic acid into inflammatory prostaglandins. In contrast to constitutive cyclooxygenase-1 (COX-1), cyclooxygenase-2 (COX-2) is induced by tissue damage during inflammatory process. Various natural products of plant origin have been shown to contain their anti-inflammatory activities via suppression of COX-2 (Jachak, 2006). Likewise, HD appeared to suppress COX-2 induction in LPS-stimulated RAW264.7 macrophage cells in a dose-dependent manner (Fig. 6). Based on this finding, $H D$ is estimated to exhibit its anti-inflammatory and antinociceptive activities via the suppression of COX-2 induction.

Although ROS at low concentrations play a role as an

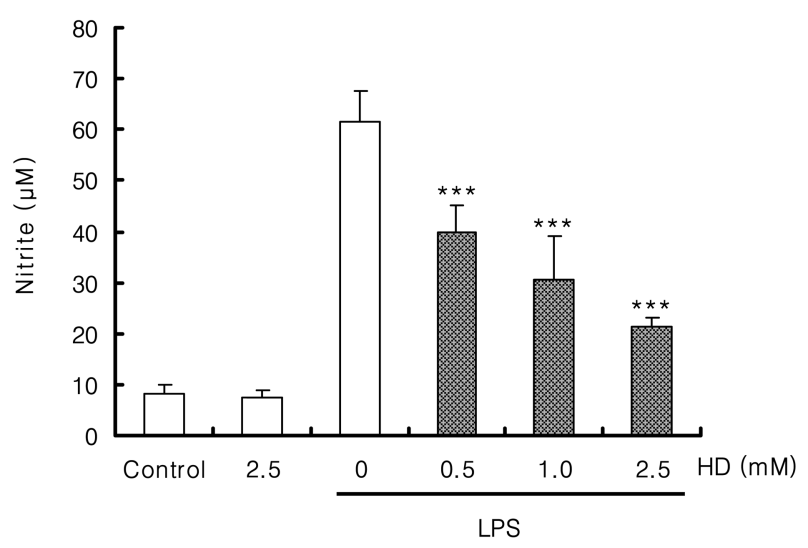

Fig. 5. Inhibitory effect of HD on LPS-induced NO production in RAW264.7 macrophage cells. The mammalian cells were incubated for $24 \mathrm{~h}$ with LPS $(1 \mu \mathrm{g} / \mathrm{ml})$ in the presence or absence of indicated concentrations of HD. Accumulated nitrite in the culture media was determined by the Griess reaction. The values are mean \pm S.E. of the three independent experiments. ${ }^{* \star *}, P<0.001$. 


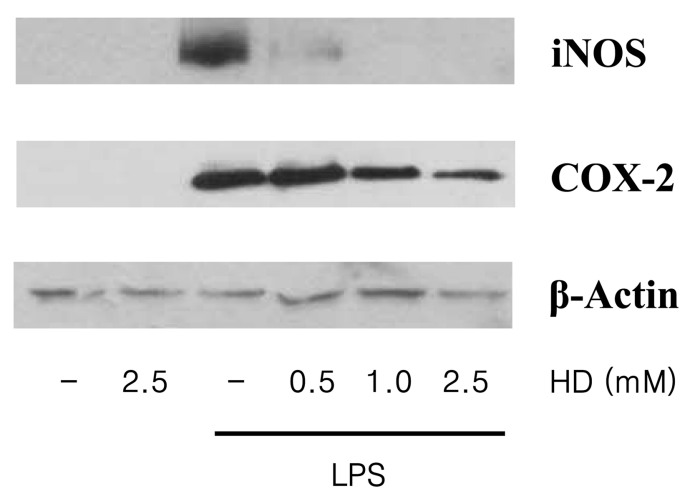

Fig. 6. Inhibitory effects of HD on LPS-induced expression of iNOS and COX-2 in RAW264.7 macrophage cells. The mammalian cells were incubated for $24 \mathrm{~h}$ with LPS $(1 \mu \mathrm{g} / \mathrm{ml})$ in the presence or absence of indicated concentrations of HD. After 24-h incubation, the cell lysates (30 $\mu \mathrm{g}$ protein) were separated by SDS-PAGE, transferred to a polyvinylidene difluoride membrane and blotted with appropriate antibodies. $\beta$-Actin was used an internal control. This blot is a representative of the two independent experiments.

intracellular messenger of various physiological events, including cell proliferation and apoptosis, the production of high amounts of ROS is cytotoxic and related with various disorders. As shown in Fig. 7, HD was able to reduce ROS level, which was elevated by LPS in the macrophages. Its diminishing effect appeared to be dose-independent. In the previous work, 4-hydroxybenzyl alcohol, an alcoholic derivative of HD, could also reduce the ROS level in the LPS-activated macrophages (Lim et al., 2007). Taken together, HD shows its anti-inflammatory

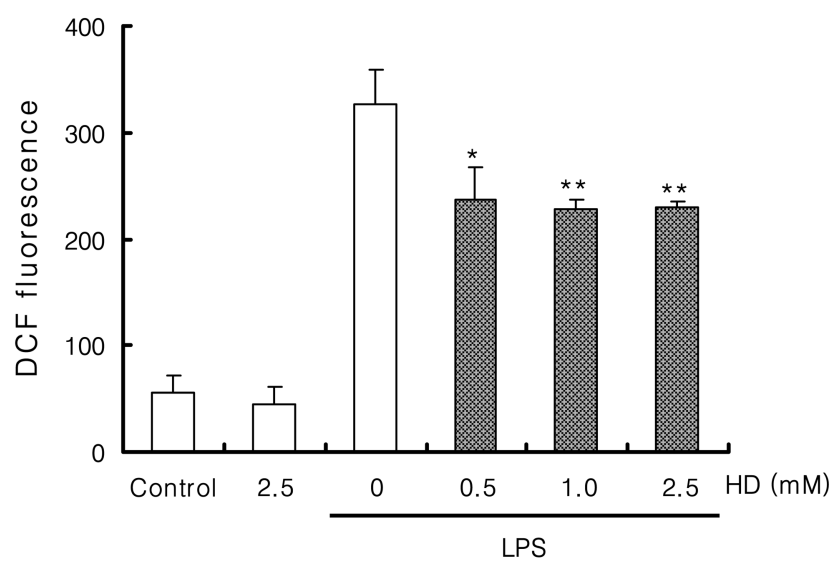

Fig. 7. Reduction of reactive oxygen species (ROS) level by HD in RAW264.7 macrophage cells activated with LPS. The mammalian cells were incubated for $24 \mathrm{~h}$ with LPS $(1 \mu \mathrm{g} / \mathrm{ml})$ in the presence or absence of indicated concentrations of HD. After 24-h incubation, the intracellular ROS levels were determined. ROS level was represented as DCF fluorescence. and anti-nociceptive activities through the reduction of NO production and COX-2 expression. Suppressing effects of HD on NO production and COX-2 expression might be subsequently based on its antioxidant activity. Although vanillin, an analogue of HD, contains anti-angiogenic, anti-inflammatory and anti-nociceptive activities, it was unable to suppress COX-2 induction in the LPS-activated macrophages (Lim et al., 2008). However, vanillin suppressed NO production and iNOS induction in the LPS-activated macrophages (Lim et al., 2008). It might be proposed that $\mathrm{HD}$ and vanillin, although their similarity in the chemical structures, act differently in the aspects of action mechanisms. The findings in this work might support the recent result that the ethanol extract of $G$ elata contains potent anti-inflammatory, anti-angiogenic and anti-nociceptic activities in in vivo and in vitro experimental models (Ahn et al., 2007). In conclusion, it has been clearly documented in this work that HD possesses some novel pharmacological activities, such as anti-inflammatory, anti-angiogenic and anti-anti-nociceptive activities, which might suggest the possible use of HD in the treatment of cancer and inflammatory diseases.

\section{REFERENCES}

Ahn, E. K., Jeon, H. J., Lim, E. J., Jung, H. J., Park, E. H. (2007) Anti-inflammatory and anti-angiogenic activities of Gastrodia elata Blume. J. Ethnopharmacol. 110, 476-482.

Chesrown, S. E., Monnier, J., Visner, G. and Nick, H. S. (1994). Regulation of inducible nitric oxide synthase mRNA levels by LPS, INF-gamma, TGF-beta, and IL-10 in murine macrophage cell lines and rat peritoneal macrophages. Biochem. Biophys. Res. Commun. 200, 126-134.

Franzotti, E. M., Santos, C. V. F., Rodrigues, H. M. S. L., Mourao, R. H. V. and Andrade, M. R. (2002). Anti-inflammatory, analgesic and acute toxicity of Sida cardiafolia L. J. Ethnopharmacol. 72, 59-65.

Ha, J. H., Lee, D. U., Lee, J. T., Kim, J. S., Yong, C. S., Kim, J. A., Ha, J. S. and Huh, K. (2000). 4-Hydroxybenzaldehyde from Gastrodia elata $\mathrm{BI}$. is active in the antioxidation and GABAergic neuromodulation of rat brain. J. Ethnopharmacol. 73, 329-333.

Ha, J. H., Shin, S. M., Lee, S. K., Kim, J. S., Shin, U. S., Huh, K., Kim, J. A., Yong, C. S., Lee, N. J. and Lee, D. U. (2001). In vitro effects of hydroxybenzaldehydes from Gastrodia elata and their analogues on GABAergic neurotransmission, and a structure-activity correlation. Planta Med. 67, 877-880.

Jachak, S. M. (2006). Cyclooxygenase inhibitory natural products: current status. Curr. Med. Chem. 13, 659-678.

Jung, J. W., Yoon, B. H., Oh, H. R., Ahn, J. H., Kim, S. Y., Park, S. Y. and Ryu, J. H. (2006). Anxiolytic-like effects of Gastrodia elata and its phenolic constituents in mice. Biol. Pharm. Bull. 29, 261-265.

Kim, H. J., Hwang, I. K., Won, M. H. (2007) Vanillin, 4-hydroxy- 
benzyl aldehyde and 4-hydroxybenzyl alcohol prevent hippocampal CA1 cell death following global ischemia. Brain Res. 1181, 130-141.

Lim, E. J., Kang, H. J., Jung, H. J., Park, E. H. (2007) Antiangiogenic, anti-inflammatory and anti-nociceptive activity of 4-hydroxybenzyl alcohol. J. Pharm. Pharmacol. 59, 12351240.

Lim, E. J., Kang, H. J., Jung, H. J., Song, Y. S., Lim, C. J., Park, E. H. (2008) Anti-angiogenic, anti-inflammatory and anti-nociceptive activities of vanillin in ICR mice. Biomol. Ther. 16, 132-136.

Olajide, O. A., Awe, S. O., Makinde, J. M., Ekhelar, A. I., Olusola, A., Morebise, O. and Okpako, D. T. (2000). Studies on the anti-inflammatory, antipyretic and analgesic properties of Alstonia boonei stem bark. J. Ethnopharmacol. 71, 179-186.

Royall, J. A. and Ischiropoulos, H. (1993). Evaluation of 2',7'dichlorofluorescin and dihydrorhodamine 123 as fluorescent probes for intracellular $\mathrm{H}_{2} \mathrm{O}_{2}$ in cultured endothelial cells. Arch. Biochem. Biophys. 302, 348-355.

Sherman, M. P., Aeberhard, E. E. and Wong, V. Z., Griscavage, J. M. and Ignarro, L. J. (1993). Pyrrolidine dithiocarbam- ate inhibits induction of nitric oxide synthase activity in rat alveolar macrophages. Biochem. Biophys. Res. Commun. 191, 1301-1308.

Singh, V. K., Mehrotra, S., Narayan, P., Pandey, C. M. and Agarwal, S. S. (2000). Modulation of autoimmune diseases by nitric oxide. Immunol. Res. 22, 1-19.

Song, Y. S., Kim, S. H., Sa, J. H., Jin, C., Lim, C. J. and Park, E. H. (2003). Anti-angiogenic, antioxidant and xanthine oxidase inhibition activities of the mushroom Phellinus linteus. J. Ethnopharmacol. 88, 113-116.

Tong, Y., Zhang, X., Zhao, W., Zhang, Y., Lang, J., Shi, Y., Tan, W., Li, M., Zhang, Y., Tong, L., Lu, H., Lin, L. and Ding, J. (2004). Anti-angiogenic effects of Shiraiachrome A, a compound isolated from a Chinese folk medicine used to treat rheumatoid arthritis. Eur. J. Pharmacol. 494, 101-109.

Vogel, H. G. and Vogel, W. H. (1997). Drug Discovery and Evaluations, Pharmacological Assays. Springer, Berlin, pp. 402403.

Whittle, B. A. (1964). The use of changes in capillary permeability in mice to distinguish between narcotic and nonnarcotic analgesics. Brit. J. Pharmacol. Chemother. 22, 246-253. 